\title{
A NOVEL METHOD OF COLOR IMAGE ENHANCEMENT BY COLOR SPACE TRANSFORMATION FOLLOWED BY GAMMA/LOGARITHMIC TRANSFORMATION
}

\author{
Karnika Dixit \\ Research Scholar \\ 476 MIG Awas Vikas Colony Fatehpur (U.P.)- 212601 \\ karnika.dixit@gmail.com \\ Mr.Kamlesh Lakhwani \\ Associate Professor \\ kamlesh.lakhwani@gmail.com
}

\begin{abstract}
Visual enhancement of image plays a very important role in the field of medical imaging. Enhanced medical images are more suitable for analysis and proper diagnosis. We present a novel method of enhancement of color medical images in this paper. We are transforming color space of the image from RGB to HIS followed by application of logarithmic and gamma transformation on saturation and intensity component respectively. Hence we obtain a visually enhanced version of the original image. We have obtained excellent color medical image enhancement results presented in this paper.
\end{abstract}

Keywords: Image enhancement, Color space transformation, Medical imaging, gamma transformation, RGB-HIS

\section{Council for Innovative Research}

Peer Review Research Publishing System

Journal: INTERNATIONAL JOURNAL OF COMPUTERS \& TECHNOLOGY

Vol 8, No. 1

editor@cirworld.com

www.cirworld.com, member.cirworld.com 


\section{Introduction}

In the last four decades, there has been a tremendous progress in the area of automation and computer-vision to aid medical professionals for better diagnosis without resorting to surgery. Medical imaging plays an important role in clinical diagnosis, therapy, medical education and researching. Magnetic Resonance Imaging (MRI), Computerized Tomography (CT), Radiography, Electrocardiogram (ECG) and Electroencephalography (EEG) etc. provide medical images containing information about patients, however, the usefulness of the information is very limited in certain cases. Recent development in computer-vision and imaging technology helps to improve quality of the color medical images resulting into much improved level of diagnosis.

Color image enhancement is a very crucial step in medical imaging and it has been attempted by many researchers before.

We present a novel method of color image enhancement based on color space transformation in this paper.

\section{Color Space Transform Enhancement}

There are two color spaces in image processing.

1. RGB Space: It is composed of three basic colors RED(R), GREEN (G) and BLUE (B).

2. HIS Space: It is composed of three basic Components i.e. HUE (H), INTENSITY (I) and SATURATION(S).

RGB is ideal for image color generation (as in image capture by a color camera or image display in a monitor screen) but it used for color description is much more limited.

HIS model is an ideal to for developing image processing algorithms based on color descriptions that are natural and intuitive to humans.
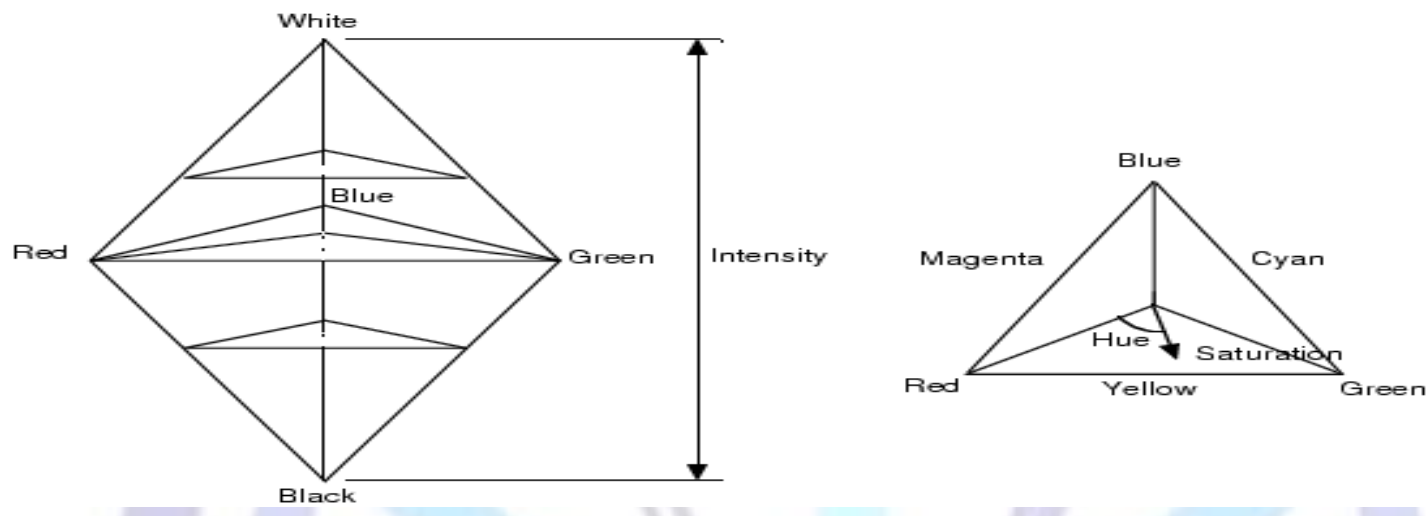

Fig-1 Relationship between the RGB and HIS color Models

During color image enhancement, although each part of RGB can be enhanced by using the method as to grey image, the hue of the image may be meaningless. Human eyes distinguish object by feeling intensity, hue and saturation of the color, not by sensing the proportion of the three basic colors, so RGB space is needed to convert to IHS space.

The three characteristics of color defined in IHS mode are intensity, hue and saturation. Saturation $S$ denotes the dark degree of color. The higher the saturation is, the darker the color is. Intensity I stand for the effect when light wave functions on the sensor. Its value is determined by the reflection coefficient. Hue $\mathrm{H}$ is the sense that the visual system feels to visible radiation object. Intensity, hue and saturation of HIS space are independent relatively. They are suitable to human's sensing. The color medical image can be further enhanced by adjusting intensity and saturation.

\subsection{RGB-HIS Transform}

In order to make advantage of RGB image, the relationship has to be built between RGB and HIS. [2]

For the given image in RGB color format, the $\mathrm{H}$ component of each RGB pixel is obtained using the equation

$$
r=\frac{R}{R+G+B}, g=\frac{G}{R+G+B}, b=\frac{B}{R+G+B}
$$

Each normalized $\mathrm{H}, \mathrm{S}$ and I components are then obtained by,

$$
\mathrm{H}=\cos ^{-1}\left\{\frac{0.5[(\mathrm{r}-\mathrm{g})+(\mathrm{r}-\mathrm{b})]}{(\mathrm{r}-\mathrm{g})^{2}+(\mathrm{r}-\mathrm{b})(\mathrm{g}-\mathrm{b})^{0.5}}\right\} \quad \mathrm{H} \in[0, \pi] \text { for } \mathrm{b}<=\mathrm{g}
$$




$$
\begin{aligned}
& H=2 \pi-\cos ^{-1}\left\{\frac{0.5[(r-g)+(r-b)]}{(r-g)^{2}+(r-b)(g-b)^{0.5}}\right\} \quad H \in[\pi, 2 \pi] \text { for } b>g \\
& S=1-\frac{3}{(R+G+B)}[\min (R, G, B)] \quad S \in[0.1] \\
& I=\frac{(R+G+B)}{3} I \in[0,1]
\end{aligned}
$$

For convenience, $\mathrm{H}, \mathrm{S}$ and I values are converted in the ranges of $[0,360],[0,100],[0,255]$, respectively.

\subsection{HIS-RGB Conversion:}

There are three sectors of interest to convert HIS to RGB.

$R G$ Sector $\left(0^{\circ} \leq \mathrm{H} \leq 120^{\circ}\right)$ : when $\mathrm{H}$ is this sector, the RGB components are given by the equation

$$
\begin{aligned}
& B=I(1-S) \\
& R=I\left[1+\frac{S \cos H}{\cos \left(60^{\circ}-H\right)}\right]
\end{aligned}
$$

And

$$
\mathrm{G}=3 \mathrm{I}-(\mathrm{R}+\mathrm{B})
$$

GB Sector $\left(120^{\circ} \leq \mathrm{H} \leq 240^{\circ}\right)$ : If the given value of $\mathrm{H}$ is in this sector, we first subtract $120^{\circ}$ from it:

$$
\mathrm{H}=\mathrm{H}-120^{\circ}
$$

Then the RGB components are

$$
\begin{aligned}
& R=I(1-S) \\
& G=I\left[1+\frac{S \cos H}{\cos \left(60^{\circ}-H\right)}\right]
\end{aligned}
$$

and

$$
\mathrm{B}=3 \mathrm{I}-(\mathrm{R}+\mathrm{G})
$$

BR Sector $\left(240^{\circ} \leq \mathrm{H} \leq 360^{\circ}\right)$ : Finally, if $\mathrm{H}$ is in this range, we subtract $240^{\circ}$ from it:

$$
\mathrm{H}=\mathrm{H}-240^{\circ}
$$

Then the RGB components are

$$
\mathrm{G}=\mathrm{I}(1-\mathrm{S})
$$




$$
\begin{aligned}
& B=I\left[1+\frac{S \cos H}{\cos \left(60^{\circ}-h\right)}\right] \\
& R=3 I-(G+B)
\end{aligned}
$$

\section{Saturation and Intensity Adjustment}

Adjusts the intensity of the colors in the image. High saturation settings boost colors and provide rich, vivid color. Low saturation settings produce pastel and more muted colors. Setting Saturation to zero produced a pure monochrome, Black and White image.

In HIS space first the intensity and Hue of the image are kept untouched, the saturation is increased. The previous saturation is replaced by the modified one. If HIS modes are retransformed to RGB mode, it is found that color of the image is becoming brighter.

\subsection{How to adjust Saturation}

To obtain a new saturation component we apply logarithmic transformation

$$
s(i, j)=c^{\star} \log (1+r(i, j))
$$

Where $r(i, j)$ is the input intensity value of the image.

\subsection{How to adjust Intensity}

Brightness is a subjective descriptor that is practically impossible to measure. It embodies the achromatic notion of intensity and is one of the key factor in describing color sensation. Keeping Hue and saturation constant, adjustment in intensity component is done by Gamma transformation.

$$
\left.s=c^{*} r(i, j) \gamma\right)
$$

The S components are enhanced by logarithmic transformations. The $\mathrm{H}$ components are not changed, because changes in the $\mathrm{H}$ components could degrade the color balance between the HSI components. The enhanced $\mathrm{S}$ and I together with $\mathrm{H}$ are converted back to RGB values. The effectiveness of the proposed method is demonstrated experimentally.
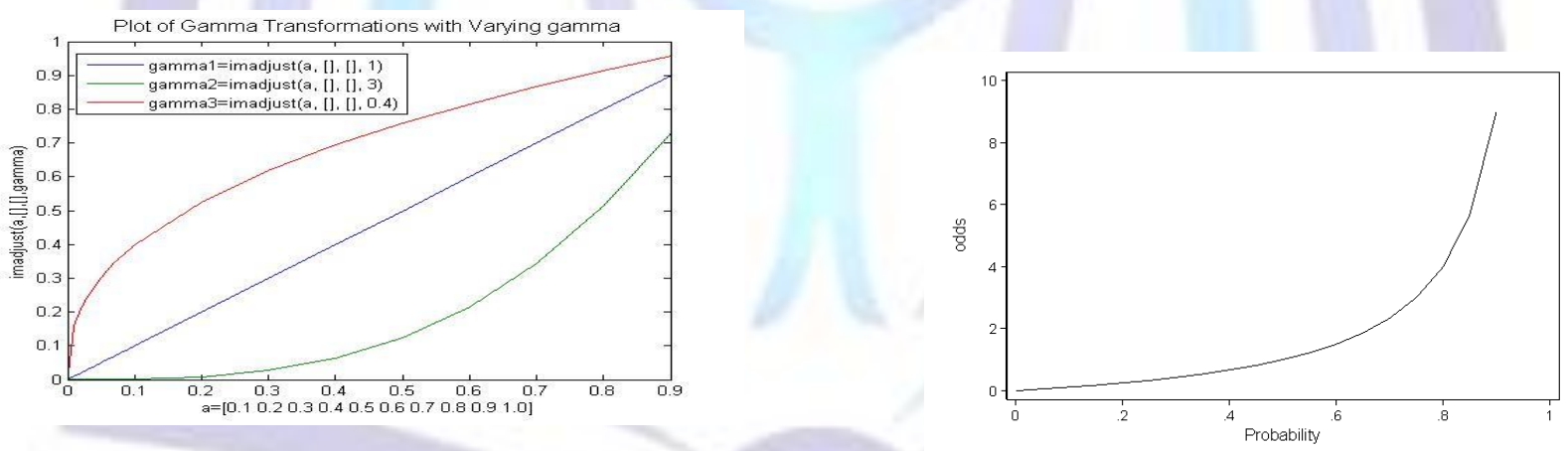

Fig-2 Curves according to gamma and logarithmic transformation.

\subsection{Proposed Algorithm:}

Step 1: The color image is transformed from RGB to HIS Space.

Step 2: Extract the H, S and I Component Separately.

Step 3: Apply logarithmic Transformation on extracted 'S'component by keeping the $\mathrm{H}$ and I component untouched.

Step 4: Concatenate the modified $\mathrm{S}$ and previous $\mathrm{H}$ and I component, to form an HIS image

Step 5: Now, Extract 'l' component, apply gamma transformation on Extracted 'l' component by keeping $\mathrm{H}$ and $\mathrm{S}$ component untouched.

Step 6: Again, concatenate the H, S and modified 'l' component.

Step 7: The image is retransformed from HIS to RGB space which results in enhanced image 


\section{Results and discussions}

We have applied the algorithm discussed in section 3.3 to the medical image shown in fig 3(a) and the enhancement result is shown in fig. 3 (b).

As you can observe that enhanced image is visually more effective for clinical diagnosis than the original image.

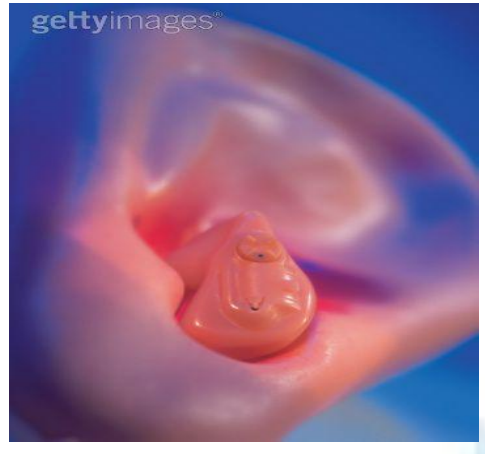

(a) Original Image

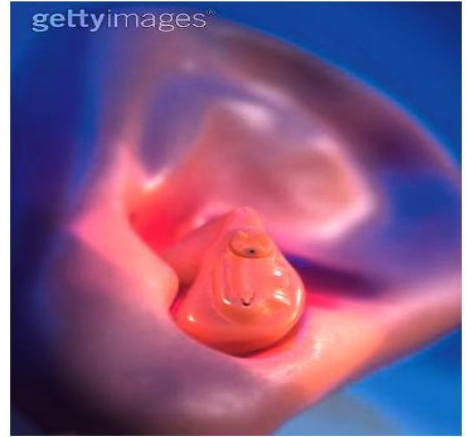

(b) Enhanced Image

Fig-3: Result of application of our method color medical image

\section{Conclusions}

We have presented a novel and very simple method for color medical imag enhancement. Computational complexity of our method is very low compared to others.

\section{References}

1. Wang Xianghong, Yang Shi-e and Xu Xinsheng "An effective method to color medical image enhancement" Complex Medical Engineering, 2007. CME 2007. IEEE/ICME International Conference, Page(s): 874 - 877 23-27 May 2007.

2. Rafael C.Gonzalez, Richard E.Woods, Steven L. Eddins, Digital Image Processing, publishing house of Electronics Industry, 2006.

3. Xingwei Wang, Lansun Shen, Danghui Liu, "A New Enhancement Arithmetic for Color Medical Image", China Biomedical Engineering Transaction, Feb. 2004.

4. Kim J K, Kim L S, "Advanced contrast enhancement using partiall overlapped sub-block histogram equalization", J. IEEE Tran. Circuits and Video Technology, vol. 11, no. 4, pp. 275-484, 2001

5. Matlab, www.mathworks.com. 